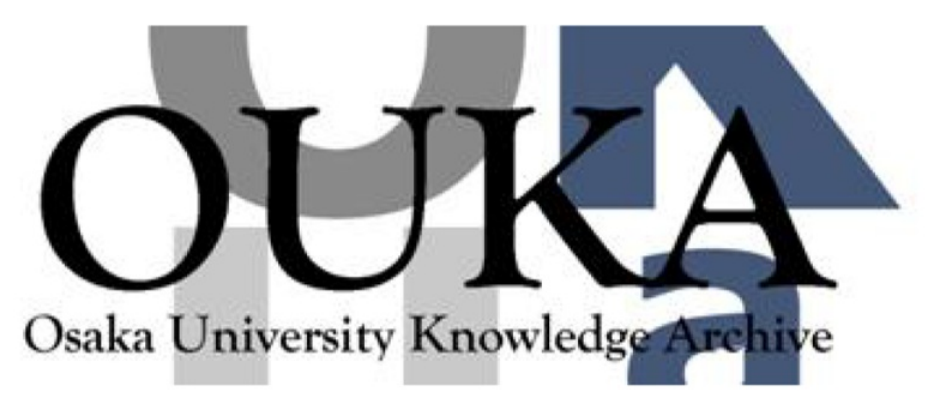

\begin{tabular}{|c|l|}
\hline Title & $\begin{array}{l}\text { Stimulated emission and lasing in } \pi \text {-conjugated } \\
\text { polymer films, microstructures and opal } \\
\text { photonic crystals }\end{array}$ \\
\hline Author(s) & $\begin{array}{l}\text { Vardeny, Z. Valy; Frolov, Sergey V. ; Chinn, } \\
\text { Douglas et al. }\end{array}$ \\
\hline Citation & $\begin{array}{l}\text { Proceedings of SPIE - the International Society } \\
\text { for Optical Eng ineering. 3797 p. 2-p. 16 }\end{array}$ \\
\hline Issue Date & $1999-12-17$ \\
\hline oaire:version & VoR \\
\hline URL & https://hdl. handle.net/11094/76942 \\
\hline rights & \\
\hline Note & \\
\hline
\end{tabular}

Osaka University Knowledge Archive : OUKA

https://ir. Library. osaka-u. ac. jp/

Osaka University 


\title{
Stimulated Emission and Lasing in $\pi$-Conjugated Polymer Films, Microstructures and Opal Photonic Crystals
}

\author{
Z.V. Vardeny", S.V. Frolov ${ }^{a *}$, D. Chinn ${ }^{a * *}$, M.N. Shkunovi***, W. Gellermann ${ }^{a}$, K. Yoshino ${ }^{b}$, A. Fujiib, \\ R.V. Gregory ${ }^{c}$, R. Baughman ${ }^{d}$, and A.A. Zakhidov ${ }^{d}$ \\ Physics Department, University of Utah, Salt Lake City, UT 84112 \\ 'Department of Electronic Engineering, Faculty of Engineering, Osaka University, Osaka 565 Yamada-Oka 2-1 Suita, Japan \\ 'School of Textiles, Fibers and Polymer Science, Clemson University, Clemson, SC 29634 \\ AAllied Signal Inc., Morristown, NJ 07962
}

\begin{abstract}
Recent studies of lasing and stimulated emission in luminescent $\pi$-conjugated polymers performed by our group are presented. Optical properties of cylindrical, high-Q, polymer microcavities are discussed. The emission spectra of pl: $: s_{1}$.c microring and microdisk lasers were measured and analyzed. Cylindrical light emitting polymer microdiodes, as possitie candidates for electrically-driven plastic lasers have been fabricated. Stimulated emission and lasing were also demonstrated for polymer solutions infiltrated in opal photonic crystals. In addition, two unusual regimes of stimulated emission characterized by narrow laser-like spectral lines were found in thin waveguiding polymer films. These regimes may be associated with random optical feedback introduced by light scattering inside the polymer films and amplified Raman scattering, respectively.
\end{abstract}

Keywords: $\pi$-conjugated polymers, stimulated emission, plastic lasers, microcavities, Raman gain.

\section{INTRODUCTION}

Organic materials continue to attract researchers as promising semiconducting media for futur electrorics Afler it recent demonstration of high optical gain and stimulated emission (SE) in films of several $\pi$-conjugated polyme : $^{1.4}$. these materials have been intensively studied worldwide as possible gain media for laser applications $s^{6.14}$. Considerable attent $n$, has been drawn to lasing in various optical cavities containing luminescent $\pi$-conjugated polymers ${ }^{9 \cdot 14}$, as well as smaller organic molecules ${ }^{15: 16}$. In our previous studies of the 2,5-dioctyloxy poly(p-phenylene-vinylene) [DOO-PPV] polymer with high photoluminescence (PL) efficiency we have demonstrated the occurrence of SE regimes, such as amplified spontaneous emission (ASE) ${ }^{8}$ and lasing?. In this contribution we summarize the optical properties of cylindrical, high-Q polymer microcavities such as microring and microdisk lasers ${ }^{14}$, and show that light emitting polymer microdiodes with cylindrical geometries are possible candidates for electrically driven plastic lasers ${ }^{14,17}$. In addition we also report on $\mathrm{SE}$ and lasing in opal photonic crystals infillusted by a variety of polymer and dye solutions ${ }^{18}$. For polymer films we also discuss two unusual SE phenomena, which le:ad to super-narrow laser-like emission lines without any cavity involved. These phenomena are random lasing ${ }^{19}$ and stimulatel Raman gain ${ }^{20}$.

There are two classes of $\pi$-conjugated polymers: luminescent and nonluminescent ${ }^{21}$. The optical properties of the luminescent polymers are very similar to those of regular organic laser dyes, such as Rhodamine or Coumarin. The primary excitations in these polymers are excitons ${ }^{22}$, which may produce PL with high quantum yield, $\eta$, defined as the ratio bet ween the number of emitted photons to the number of absorbed photons ${ }^{23}$. Typically. the radiative decay channel of excitons has to compete with various channels of nonradiative decay; as a result, $\eta$ is always less than unity. In our studies we have focused on tivo varieties of luminescent conducting polymers with particularly high PL yields, namely dioctyloxy-PPV (DOO-PPV) $)^{2 i}$ with $\eta \approx 0.2$ and poly(1-phenyl-2-p-n-butylphenylacetylene) [PDPA-nBu] with $\eta \approx 0.4$. Their backbone structure. optical absorption and PL spectra are shown in Fig. 1. The Stokes-shifted PL bands, which are broadened by both homogeneous (phonons) and inhomogeneous (disorder) contributions, appear in the spectral range where the ground state absorption is weak. Therefore, 


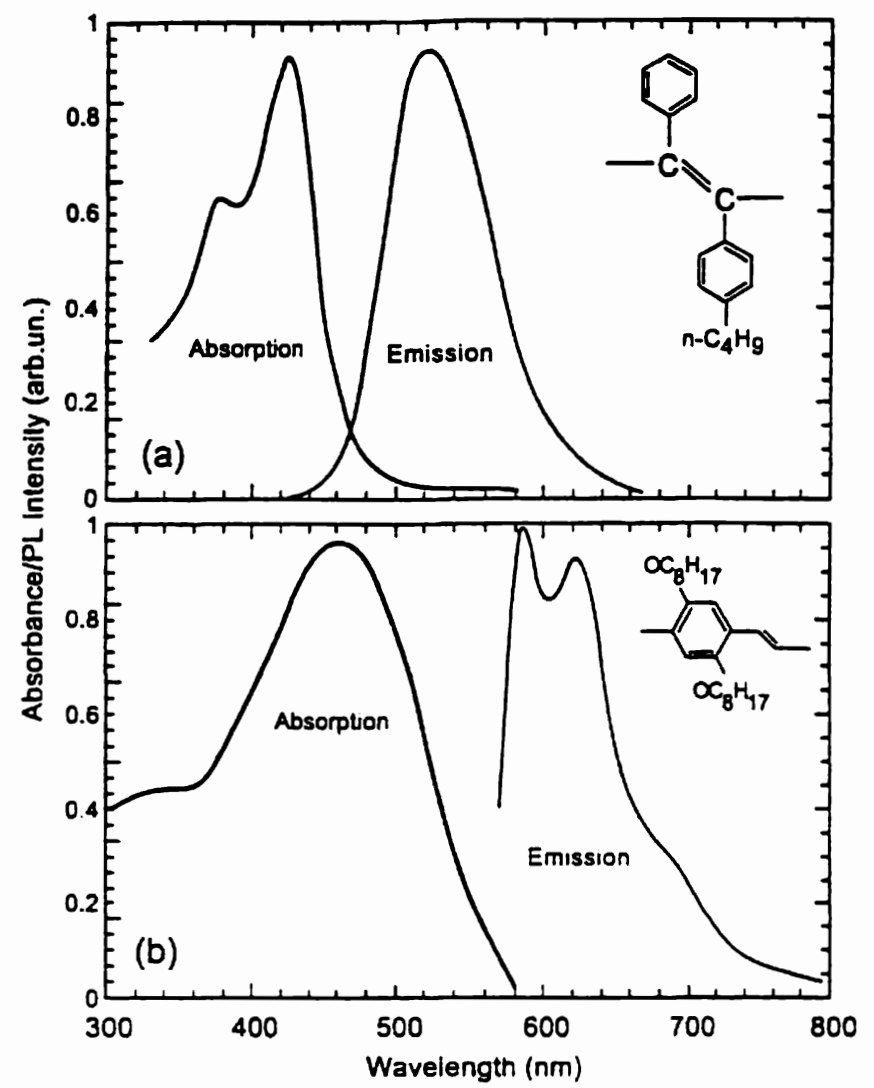

units

a simple exciton model has been adopted to describe the optical transitions responsible for absorption and emission in these polymers, as shown in a configuration coordinate diagram of Fig.2(a). According to this model, the lowest excitations in $\pi$ conjugated polymers form a 4-level system. where transitions $l \rightarrow 2$ and $3 \rightarrow 4$ describe the absorption and emission processes. respectively. The phonon-assisted relaxation transitions $2 \rightarrow 3$ and $4 \rightarrow 1$ are very efficient and occur within $1001 \mathrm{fsec}$ and $1 \mathrm{psec}$, respectively. On the other hand, the exciton lifetime, i.e. the decay (mostly nonradiative) time of level 3 , in our polymer films is in the range of $100 \mathrm{ps}$ to $1 \mathrm{~ns}$, as illustrated in Fig.2(b). We conclude that it is possible to achieve inversion between the levels 3 and 4 and thus satisfy the main requirement for a laser medium.

The polymer residual absorption, $\alpha_{\text {res }}$, at the optical frequency between levels 3 and 4 determines the threshold excitation density that is necessary for the population inversion and consequently, lasing. It is preferable, therefore, to have $\alpha_{\text {ris }}$ as low as possible. However, one of the primary requirements for a polymer laser medium is its high PL yield. It has been noticed that in general, $\eta$ is determined not only by the exciton lifetime, but also by the exciton generation yield. The later may also te less than unity due to the formation of interchain excitations ${ }^{25}$ and other nonradiative species. It is essential, therefore, for achieving high $\eta$ to have both long exciton lifetime and low generation yield of nonemissive excitations. The last fundamental condition influencing the performance of a polymer as a SE medium is the spectral extent of the excitonic photoinduced absorption (PA) in photopumped lasers, and current induced absorption (CIA) of polarons in electrically driven lasers. The PA or CIA spectrum may overlap with the spectrum of the stimulated emission (SE) and thus cancel the total optical gain. It has been shown that the PA and SE spectra of singlet excitons in DOO-PPV are well separated ${ }^{22}$, indicating that this polymer can be a good candidate for photopumped laser media.

\section{EXPERIMENTAL}

For studies of SE in polymer films, DOO-PPV and PDPA films with uniform thickness, $d$, ranging from $0.5 \mu \mathrm{m}$ to $+\mu \mathrm{m}$ were slowly spin-coated from fresh chloroform solutions onto quartz substrates. The variation in $d$ was typically less than $5 \%$ over 


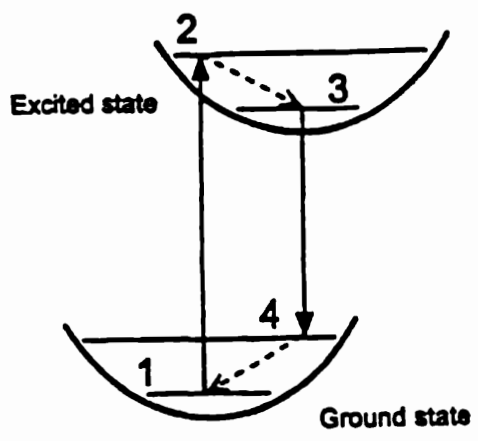

(a)

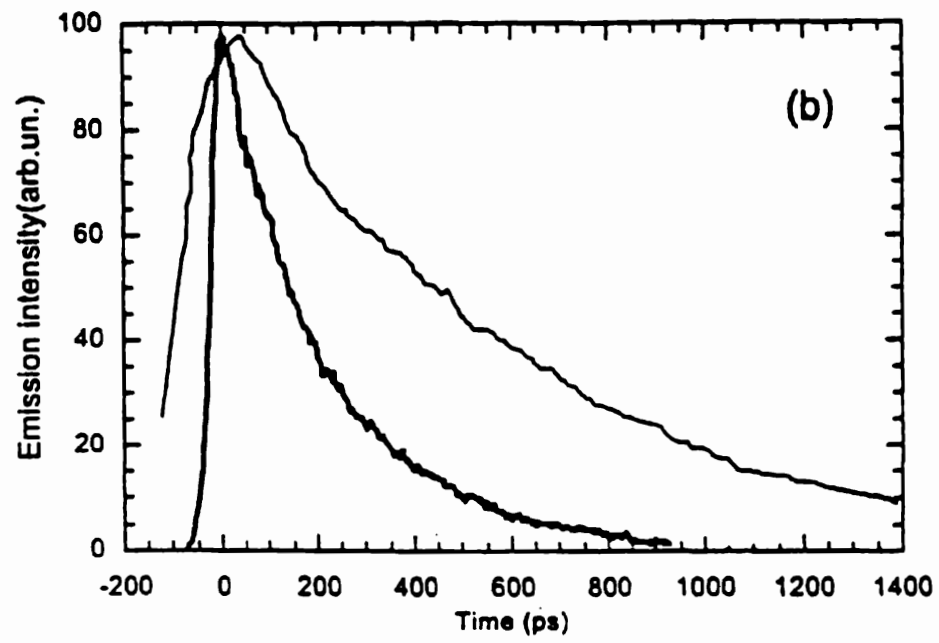

Fig.2. (a) Configuration coordinate diagram of energy levels (numbered 1 through 4 ) in luminescent conducting polymers; solid arrows indicate optical transitions corresponding to absorption and emission processes, respectively. (b) Time-resolved photoluminescence in DOO-PPV (thick line) and PDPA-nBu (thin line) films.

the film length of $1 \mathrm{~mm}$. The excitation source for the films was a regenerative laser amplifier as described below. The pump laser beam was focused on the polymer film using a stripe geometry. The length of the stripe-like excitation arca could be varied from $100 \mu \mathrm{m}$ to $6 \mathrm{~mm}$ using a variable-width slit in front of the film, which could block parts of the pump beam. The polymer emission was collected from either the front or the side of the substrate and spectrally analyzed using either a 0.25 meter spectrometer or a 0.6 meter triple spectrometer. All experiments were performed in dynamic vacuum at room temperature.

In our studies of lasing in polymer films, we again used soluble derivatives of both PPV and PDPA: DOO-PPV and PDPA-nBu respectively. Cylindrically shaped thin polymer films were prepared by dipping commercially available optical fibers into saturated chloroform solutions. Thin polymer rings were consequently formed around the glass cylindrical cure following the fast drving in air. The estimated thickness of the deposited polymer rings was about $2.3 \mu \mathrm{m}$. The excitation source was the Nd:YAG regenerative laser amplifier producing 100 ps pulses at a repetition rate of $100 \mathrm{~Hz}$. This laser light was either frequency doubled $(532 \mathrm{~nm})$ or tripled $(355 \mathrm{~nm})$ for pumping DOO-PPV or PDPA-nBu films, respectively. The pump beam was focused using a cylindrical lens into a $100 \mu \mathrm{m} \times 5 \mathrm{~mm}$ stripe perpendicular to the polymer coated fiber $(\varnothing 10-200 \mu \mathrm{m})$; thus only a small fraction of the pump light was absorbed by the polymer film. The pump beam polarization could be rotated to be either parallel or perpendicular to the fiber axis. The light emitted from the excited polymer ring was collected in the plane of the ring with a round lens and spectrally analyzed using a 0.6 meter triple spectrometer and a CCD array with spectral resolution of about $1 \AA$,

Micro-crystalline opals with crystal sizes of 20-100 $\mu \mathrm{m}$ were prepared from crystallizing colloidal suspensions of nearly monodisperse $\mathrm{SiO}_{2}$ spheres with diameters $d$ varying between : $90 \mathrm{~nm}$ and $300 \mathrm{~nm}$, as described elsewhere ${ }^{26}$. A typical opal slab 
size was $1 \mathrm{~mm} \times 1 \mathrm{~cm} \times 1 \mathrm{~cm}$. After the complete penetration of the solution, the opals became semitransparent due to close matching between the refractive indices of solvents and silica $(\Delta n=0.01)$. As a result, light scattering from the silica nanoballs was relatively weak: from transmission measurements in the spectral range between 550 and $650 \mathrm{~nm}$ for opals infilurated with ethylene gloycol we estimated the mean photon diffusion length, $l^{*} \geq 0.5 \mathrm{~mm}^{18}$. We found that Bragg scan aring stop bands which are known to exist in opals did not influence the SE spectra. The opal slabs soaked in solutions containing gain media (either R6G or DOO-PPV) were placed inside a $1 \mathrm{~cm} \times 1 \mathrm{~cm}$ quartz cuvette and photoexcited using the previously described experimental setup ${ }^{18}$. The strip-like excitation at intensities above the SE threshold resulted in the emission from the side of the slab of $a \sim 5^{\circ}$ divergent beam directed along the stripe axis.

\section{LASING}

\subsection{Cylindrical microcavities}

True lasing has been first demonstrated in a Fabry-Perot resonator using a dilute solution of MEH-PPV", a poly mer with optical characteristics similar to those of DOO-PPV ${ }^{22}$. Analogous results have been obtained later with solutions of other $\pi$-conjugated polymers ${ }^{28}$. Early time-resolved studies have shown that unlike laser dyes, conducting polymers do not experience concentration quenching and, therefore, may exhibit optical amplification, or gain, even when they are prepared as thin films ${ }^{29}$. However, because the absorption length in neat, undiluted polymer films is much shoner than that in solutions, it is much more difficult to use thin films as gain media in open laser cavities formed with external mirrors ${ }^{10.12}$. Thus work in this area has mainly concentrated on microstructures, such as planar ${ }^{130}$ and cylindrical microcavities ${ }^{14}$. distributed feedback lasers ${ }^{13.31}$ and other configurations using waveguiding films.

The microring and microdisk cavity structures are schematically shown in Fig.3. In both cases a thin. uniform polymer film forms the entire cavity of the laser. The main advantage of such microlasers is the ease with which they can be produced. particularly the microring lasers. Typically, an optical fiber was dipped into a saturated chloroform solution of DOO-PPV or PDPA-nBu. which after quick evaporation uniformly coated the fiber and produced a complete cylinder of $\sim 1 \mu \mathrm{m}$ in thickness, $100 \mu \mathrm{m}$ or more in length and a diameter. $D$. predetermined by the size of the fiber. Alternatively. any other cylindrical substrate could be used with equal success. e.g. lasing was demonstrated using metal wires and also polyaniline fibers ${ }^{14}$. The fabrication of microdisks was slightly more difficult: thin spin coated films were photo-lithographically etched to produced microdisk arrays of various diameters. The substrate was usually either quarz or indium tin oxide (ITO) coated glass. The full description of microlaser fabrication and the experimental setup is given elsewhere ${ }^{14}$.

An important advantage of a cylindrical microcavity is its relatively high finesse, or quality factor $\mathrm{Q}^{\circ}$. The light in such cavities is confined inside the gain medium by total internal. practically lossless reflections: the radiation leakage is due to the cavity surface curvature and light scattening from imperfections. In companson a planar microcavity always experiences losses due 10 imperfect reflections from the two highly reflective mirrors forming the microcavity ${ }^{120}$ Optical modes inside a cylinder are given by the solution of the two-dimensional Helmholtz equation ${ }^{33}$, which lead to longitudinal modes satisfying the equation:

$$
M \lambda_{M}=2 \pi R \pi_{e F}
$$

where $R$ is the $\mu$-cavity radius, $\pi_{e f}$ is the effective mode refractuve index and $M$ is the mode index. The laser modes are also classified by another index. N. according to their radial intensity distribution inside the disk ${ }^{32}$.

Eq. (1) in fact describes the longitudinal modes of a ring resonator formed by the thin polymer waveguide witl, the total length of $2 \pi R$ TE modes (polarization is in the plane of the waveguide and parallel to the fiber axis) with $N=1$ hilve the highest $Q$ and thus the lowest threshold intensin. $I_{0}$. These modes may dominate the spectrum of the mucroring laser for very thin polymer films. which can be seen in fig.t(a) (see also ref. 9). From Eq (1) we obtan an expression for the intermodal separation, $\Delta \lambda$ $=i_{M-1}-i_{M}$

$$
\Delta \lambda=\frac{\dot{\lambda}^{2}}{2 \pi \mathrm{Rn}_{\mathrm{eff}}}\left(1-i \frac{\hat{\partial \mathrm{n}_{\mathrm{ef}}}}{\hat{o} \dot{\lambda}}\right)^{-1}
$$




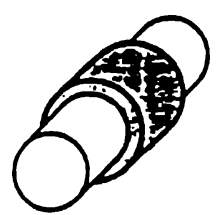

(a)

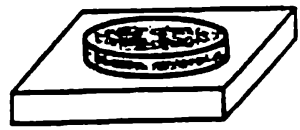

(b)

Fig.3. Plastic cylindrical microcavities: (a) microring and (b) microdisk.

Assuming negligible dispersion in Eq.(2), from Fig.4(a) we find that for PDPA-nBu films $n_{-} \approx 1.75$. We note that the spectrum in Fig.4(a) may be used to determine $\partial n_{d} d \partial \lambda$, if $n_{0}$ can be measured independently.

The cavity $Q$ factor can be generally defined as $Q=\omega t_{c}$, where $\iota_{c}$ is the decay lifetime of a cavity mode ${ }^{33}$; thus the longer is the photon lifetime inside the cavity, the higher is $Q$. The value of $Q$ may be influenced by various contributions a $1 d$ near the laser threshold it is given by ${ }^{13,19}$.

$$
Q^{-1}=Q_{a v a}^{-1}+Q_{\text {carl }}^{-1}+Q_{a b x}^{-1}
$$

where $\mathrm{Q}_{\mathrm{cav}}{ }^{-1}$ describes radiation losses determined by the cavity geometry for a given mode, $\mathrm{Q}_{\text {scat }}{ }^{-1}$ is due to scattering from imperfections inside and on the surface of the cavity, and $\mathrm{Q}_{\mathrm{abs}}{ }^{-1}$ is determined by self-absorption of a cold gain medium according to $\mathrm{Q}_{\mathrm{abs}}=2 \pi n / \alpha_{\text {res }} \lambda$, where $n$ is the refractive index and $\alpha_{\text {res }}$ is the absorption coefficient at the emission wavelength $\lambda$. $Q_{c a v}$ is known to strongly depend on $\mathrm{M}$ and $\mathrm{N}^{34}$. It is maximized for $\mathrm{N}=1$ and the corresponding modes are called whispering gallery modes, for which at $M>20$ we find that $Q_{a a r}>10^{432}$. In our measurements of both microring and microdisk lasers made from pristine polymer films, we have found ${ }^{9.14}$ that typical $Q$ values are on the order of $3 \cdot 10^{3}$. We have concluded that despite rather high $Q_{\text {cav }}$ values, $Q$ of a polymer microcavity is usually limited by scattering losses and material absorption, $Q_{\text {scat }}{ }^{-1}$ and $Q_{\mathrm{abs}}{ }^{-1}$, respectively. $Q_{\text {scat }}^{-1}$ can be somewhat minimized by making smoother microactivity surfaces and purifying the polymer solution. $Q_{\text {obs, }}$ on the other hand, is determined by $\alpha_{\text {ses }}$ and thus is difficult to change. However, it is possible to dilute the polymer by mixing it with other, less lossy and optically neutral media, which may lower $\alpha_{\text {res }}$ and, therefore, increase $Q_{\text {abs. }}$ This has been successfully demonstrated with various blends of organic dyes, oligomers ${ }^{15,16}$ and polymers ${ }^{10,12}$.

\subsection{Microring and microdisk lasers}

Fig.4(a) shows the emission spectra obtained from a PDPA- $n$ Bu microring deposited on a fiber with $D=125 \mu \mathrm{m}$. The broad, featureless PL band at low excitation intensities, 1 . is transformed into a multimode ring laser spectrum at higher $I$; this transition into the lasing regime is accompanied by a kink in the emission intensity vs. I dependence at the threshold excitation intensity. $J_{0}^{9.14}$. The wavelength of each laser mode. $\lambda_{M, N}$, is given by the solution of Eq.(1).

Fig.4(b) shows the emission spectra of a DOO-PPV microring laser with $D=20 \mu \mathrm{m}$. These spectra cannot be adequately described by Eq.(1). since more than one set of longitudinal modes is observed. However, it is possible to nıodel them using two lowest order TE modes with $N=1$ and $N=2$ (TM modes were nol observed in thin microrings). As a result of such modeling, the $M$ and $N$ index numbers may be assigned to each laser line, as shown in Fig.4(b). The only fitting parameter is $n_{\text {on }}$ which we find (assuming near zero dispersion) to be 1.68 and 1.677 for $N=1$ and $N=2$, respectively. Higher order waveguided modes (with $\mathrm{N}=2$ ) are expected to have lower $n_{\text {ef }}$ due to their deeper penetration inside the glass fiber.

In order to avoid light propagation inside the optical fiber, we used thin metal wires as a cylindrical core for the microring ${ }^{14}$. Although an absorptive metal surface may quench stimulated emission and prevent lasing, a thicker (>5 $\mu \mathrm{m})$ pclymer film helps to isolate the modes from the metal core and thus minimize optica' losses ${ }^{14}$. Fig.5(a) shows the emission spectra obtained from 


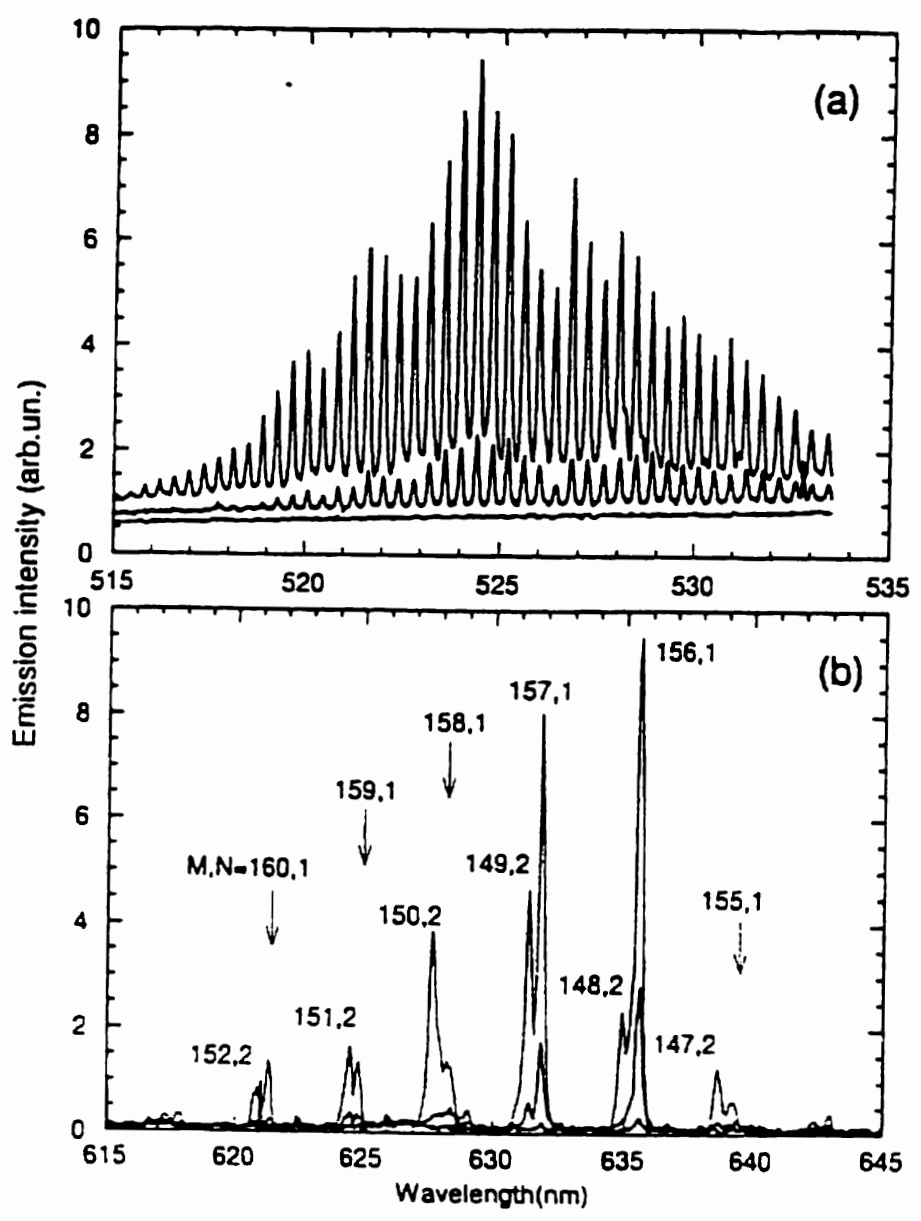

Fig. 4 (a) Emsssion spectra from a PDPA-nBu mucronng on a $0125 \mu \mathrm{m}$ optucal fiber at difierent excitation intensities; excitation energses from top to bottom are $\lfloor\mu \mathrm{J}, 0.7 \mu \mathrm{J}$ and $0.6 \mu \mathrm{J}$, respectively. (b) Emssion spectra from a DOOPPV micronng un a $020 \mu \mathrm{m}$ fiber. excitation energles are $165 \mathrm{NJ}, 90 \mathrm{NJ}$, and $65 \mathrm{~J}$, respectively. $M$ and $N$ indices are assigned to each laser line (see iexl).

a DOO-PPV microring $(D=35 \mu \mathrm{m})$ deposited on a $025 \mu \mathrm{m}$ aluminum wire. Al low excitation intensities the spectrum is dominated by a single set of equidistant longitudinal modes; however. it can be seen from Fig.5(a) that at higher intensities additional modes with a higher threshold appear in the emission spectrum. Assuming negligible dispersion. from $\Delta \lambda$ of Fig.5(a) we calculate using Eq. (2) $n_{e f f}=2.23$. which is significantly higher than the value estimated from Fig.4(b), where $n_{f o}=1.7$. Lower $n_{e f f}$ for the thinner microrings on glass fibers indicates that the lasing modes in these cavities are not confined to the polymer film. but also partly propagate inside the glass core, where the refractsve index is low $(\sim 1.4)$. The modes in thick microrings, however. are fully contained inside the polymer and therefore have higher $n_{e f}$.

We found that polymer microdisk lasers behave similarly to thick microring lasers. Typically, a single microdisk with a diameter ranging from $8 \mu \mathrm{m} 10128 \mu \mathrm{m}$ was photoexcited by a focused laser beam from the Nd:YAG laser amplifier, producing $100 \mathrm{ps}$ or $10 \mathrm{~ns}$ pulses at $532 \mathrm{~nm}$. The polymer emission was collected in the plane of the microdisk using the same expenmental setup used for the microring laser measurements. Unlike microrings, however, microdisks provide good lateral confinement for the lasing modes. In addition. it is easy 10 achieve a complete and uniform excitation of the whole microdisk area. The mode structure of the disk microcavity is also described by Eq.(1). In fact, the spectra of the microdisk lasers are vinually indıstunguishable from those of micronings, as shown in Fig.5(b). Using Eq. (2) and again assuming zero dispersion for the DOO-PPV microdisk laser shown in Fig. $5(\mathrm{~b})$ with $D=16 \mu \mathrm{m}$, we calculate $n_{e f f}=2.22$, which is close to $n_{e f}$ obtained for thick DOO-PPV microring deposited on metal wires. 


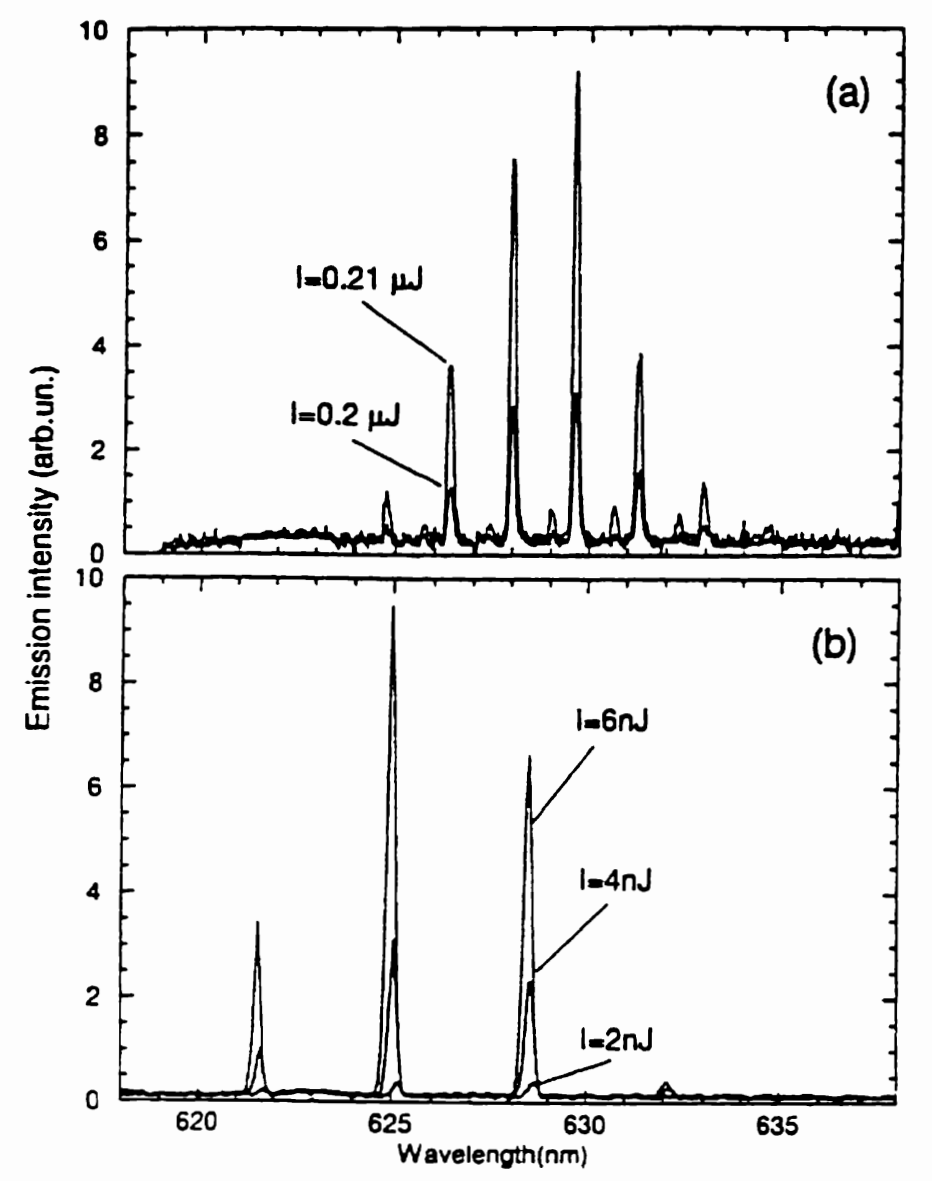

Fig.5. (a) Emission spectra from a DOO-PPV microning with $D=35 \mu \mathrm{m}$ or a $025 \mu \mathrm{m}$ Al uire at two different excitation intensities; (b) Emission spectra from a thun DOO-PPV microdisk with $\mathrm{D}=16 \mu \mathrm{m}$ at three different excitation intensities as indicated.

The emission spectra of polymer microlasers shown in Figs. 4 and 5 were obtained using 100 ps pulse excitation. Although the duration of such excitation is much longer than the photon lifetime in the microcavity ( $(L=Q / \omega \sim 1 \mathrm{ps})$, it is on the order of the exciton lifetime in the polymer film and therefore may not be considered quasicontinuous ${ }^{35}$. We studied the effects of longer pulse excitation and found that the 10ns-excitation also resulted in efficient lasing. In Fig. 6 we compare the cmission spectra obtained from a single DOO-PPV microdisk $(D=32 \mu \mathrm{m})$ using $100 \mathrm{ps}$ pulses (a) and 10 ns pulses $(\mathrm{b})$. The mode structures in both cases are essentially identical. The main difference is the pronounced broadening and blue-shift of the laser lines in the case of 10 ns excitation. The blue-shift may still be observed with 100 ps pulses. Both of these effects are highly detrimental 10 the performance of any laser. We speculate that this might be due to excessive heating of the polymer film, since most of the excitation energy is spent on heating of the polymer film and the longer pulse excitation does provide more energy. The spectral blue-shift (decrease of $\lambda_{M}$ ). however. indicates that either $D$ or $n_{e f}$ decreases as I increases. and this cannot be explained by an increase in temperature. We thus speculate that the blue-shift is caused by lowering of the polymer refractive index at high excitation densities Also. the substantial line broadening in the case of long pulse excitation can be attributed to the reduction of the $\mathrm{Q}$ facior. which may be due to either microcavity deformations caused by overheating. or additional absorption losses from triplet exciton population build-up 22 .

\section{CYLINDRICAL MICRO-LEDS}

Our group has been simultaneously engaged in studying micro-LEDs with cylindrical geometry ${ }^{1736}$. As pointed out above, microlasers with cylindrical geometry may have a high $Q$ that is limited only by $\alpha_{\text {res }}$ of the gain medium. We have therefore fabricated a variety of cylindrical micro-LEDs and studied their properties at low current densities with the aim of achieving laser action when using current injection at much higher density in a pulsed current regime. 


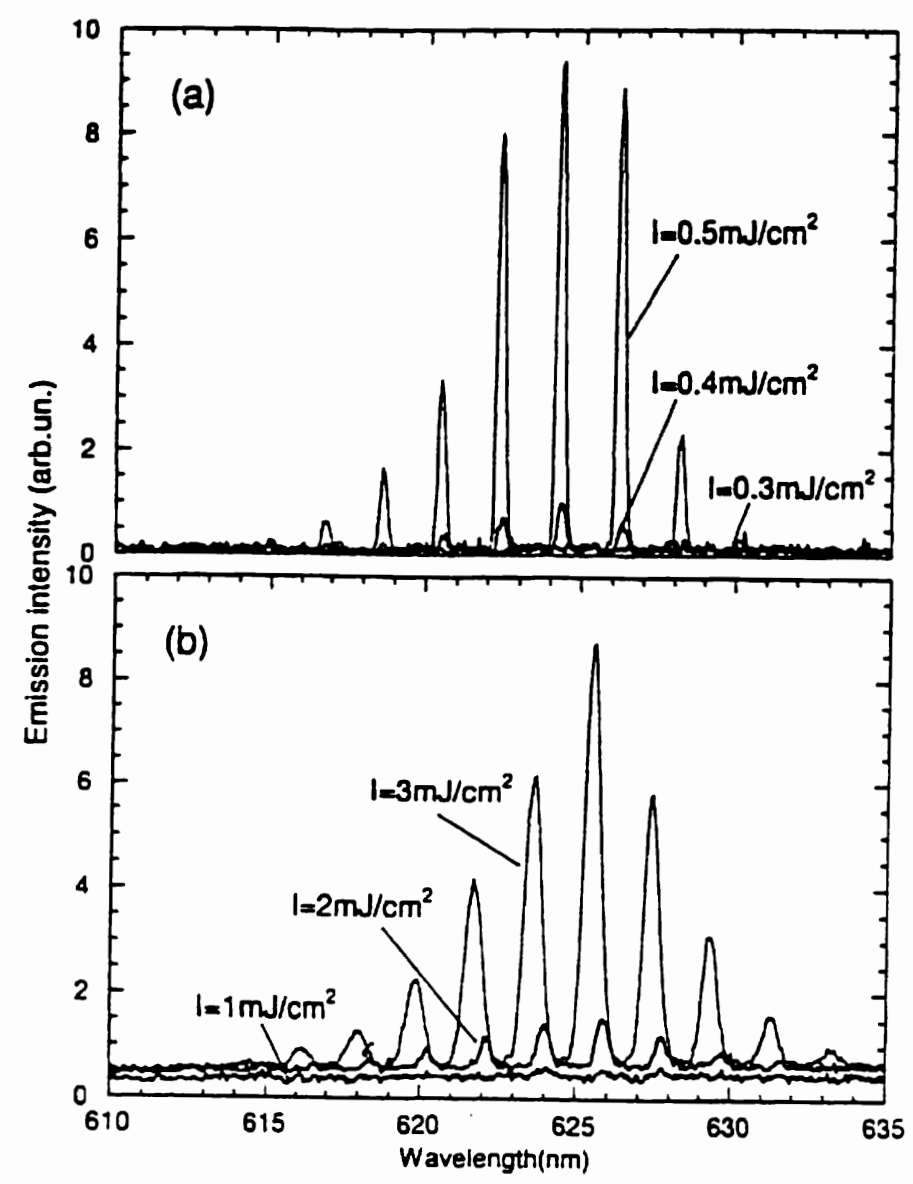

Fig.6. Emission spectra of a DOO-PPV mictodisk with $D=32 \mu \mathrm{m}$ at different excitation intensitues using (a) 1 no ps pulse excitation and (b) $10 \mathrm{~ns}$ pulse excitation.

Two kinds of cylindrical micro-LED devices were studied: microdisks and microrings. as shown in Fig.7(a). For microdisks. we deposited DOO-PPV or PDPA-nBu thin films on good quality ITO substrates for hole injection. The film was then capped with an Al metal layer or electron injection. followed by etching of both polymer and Al layers to produce pattemed microdisks of various diameters. For microrings. we coated ITO on optical fibers of various diameters. which were drawn out from commercial multimode silica fibers. ITO layers with typical thickness of $200 \mathrm{~nm}$ and sheet resistance of $10 \Omega / \mathrm{cm}$ were prepared by an electron beam evaporation process. Thin polymer films with estimated thickness of $1 \mu \mathrm{m}$ were then dr.posited onto the ITO coated fibers by dipping the fibers into saturated toluene solutions of DOO-PPV. Afterwards, a semitransparent Al layer was deposited in high vacuum of $10 \mathrm{~Pa}$. The length of the Al electrode was about $2 \mathrm{~mm}$.

The electrical properties of the polymer LEDs, such as current-voltage (I-V) characteristics, were measured using a Keithley 236 source measuring unit connected 10 a personal computer. The emission intensity in the forward biasing mode was detected using a photomultiplier. The emission spectrum was measured using a triple spectrometer and an optical multichannel detection system with a liquid nitrogen cooled charge coupled device with $-0.3 \mathrm{~nm}$ resolution.

Typical I-V and voltage-emission intensity (V-L) characteristics at forward biasing of a $0125 \mu \mathrm{m}$ micronng LED with DOOPPV polymer are shown in Fig.7(b). The LEDs exhibited nypical rectifying characteristics. in which the ITO and Al layers act as hole and electron injecting electrodes. respectively. The emission onset is seen to occur at about 9 Volts, but we found that the driving voltages increase with the polvmer thickness. As evident in the I-L plot shown in Fig. 7(c), L increases monotonously with I. similariy to DOO-PPV flat-type LEDs.

The electroluminescence (EL) spectrum of the microring LED is shown in Fig.8. As evident from this figure, the EL spectrum is similar to the PL spectrum. It has a broad double hump feature; lasing modes could not be obtained since the injected current 

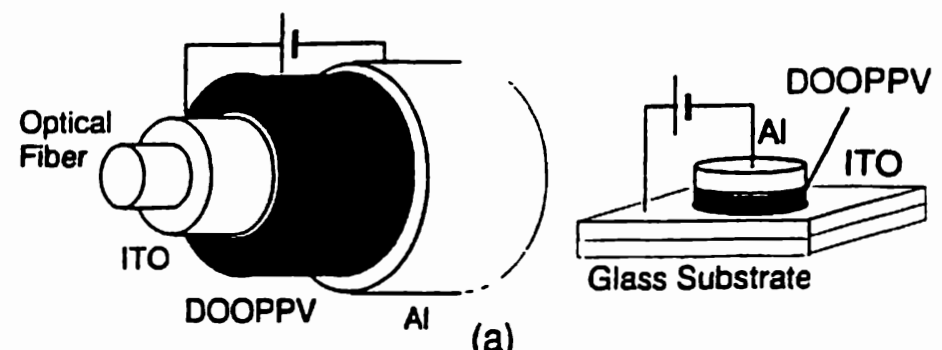

(a)
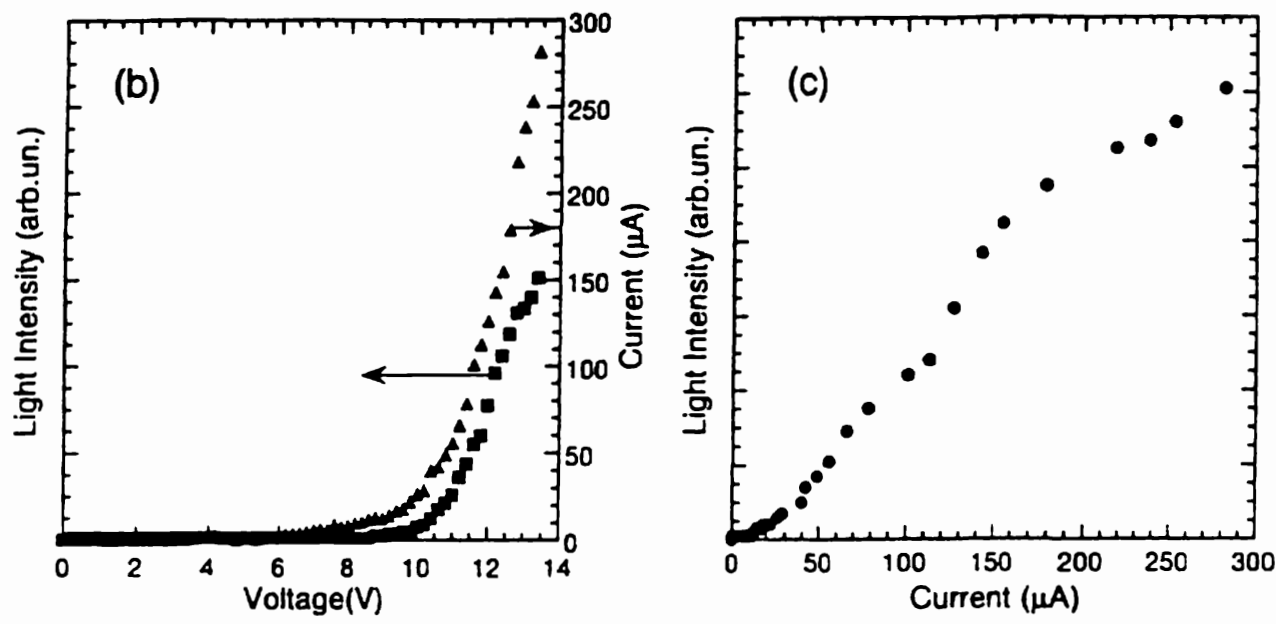

Fig. 7 (a) Microring and microdisk LED devices. (b) Emission-Voltage and Current-Voltage characteristics of a $0125 \mu \mathrm{m}$ mucroring LED with DOO-PPV polymer, (c) Emission-Curtent characterisitc of the microning LED.

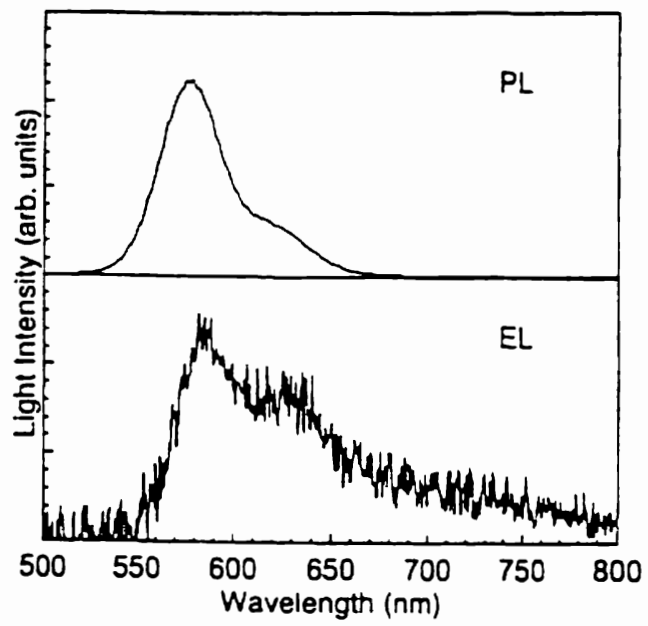

Fig. 8 Electroluminescence spectrum of the DOO-PPV microning LED

density was only about $100 \mathrm{~mA} / \mathrm{cm}^{2} \mathrm{DC}$. We also achieved bright EL from a microdisk LED using up $102 \mathrm{~A} / \mathrm{cm}^{2} \mathrm{DC}$ current density ${ }^{-x}$. In order to produce a current-driven organic laser, it will be necessary to use pulsed current sources. We have determined ${ }^{14}$ that laser action is possible with sub- $\mu$ sec light pulses $(<200 \mathrm{~ns})$. We may then assume that sub- $\mu$ sec electrical pulses will be sufficiently shor for electrically driven polymer laser diodes. From the laser threshold intensity for our photopumped microlasers. neglecting the polaron absorption and assuming a $5 \%$ internal quantum efficiency, we estimate the threshold current density necessary for lasing to be on the order of $10^{4} \mathrm{~A} / \mathrm{cm}^{2}$. Such current densities are within the present limits of polymer LEDs, where current densities as high as $10^{6} \mathrm{~A} / \mathrm{cm}^{2}$ have been recently demonstrated ${ }^{37}$. 


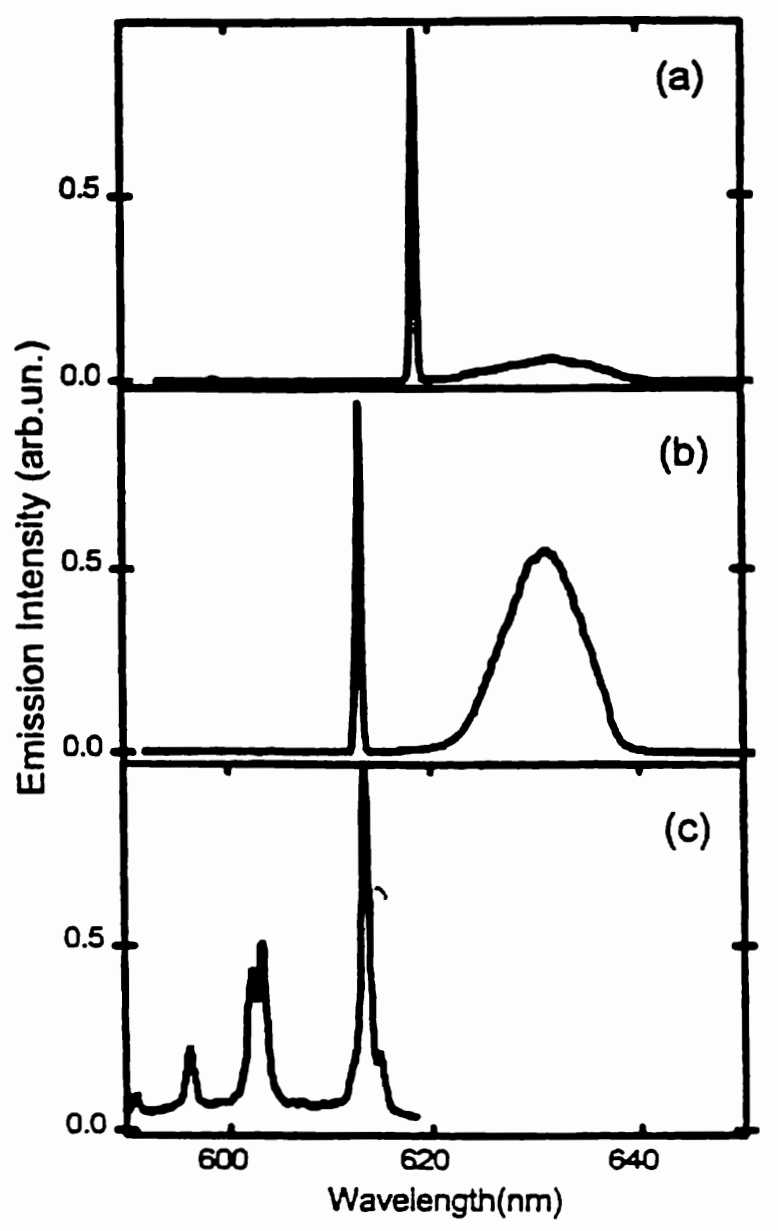

Fig.9 Emission spectra from a DOO-PPV film excited (a) with $90 \mu \mathrm{J}$ pulses at $563.5 \mathrm{~nm}$ and (b) with $12 \mu$ pulses at $559 \mathrm{~nm}$; (c) spontaneous Raman scattenng spectum of a DOO-PPV film measured using $752 \mathrm{~nm}$ excitation and plotted vs. wavelength assuming h!pothetical 559 nm excitation.

\section{AMPLIFIED RAMAN SCATTERING}

An interesting phenomenon that results in narrow laser-like lines even in the absence of an optical cavity is the resonant amplification of the most strongly coupled Raman modes in the gain medium ${ }^{20}$. Figs.9(a) and $9(\mathrm{~b})$ demonstrate this type of optical amplification in a thin film of DOO-PPV. The excitation source for these measurements was a Raman-shifted 100 pspulsed laser tuned at $563.5 \mathrm{~nm}$ (a) and $559 \mathrm{~nm}$ (b). respectively, both of which cover the long wavelength ta:l of the polymer absorption band. In both cases the regular ASE band centered al $-630 \mathrm{~nm}$ did not shift with the excitation wavelength. However. a very intense, narrow emission line appeared in both cases on the high energy side of the ASE band. We determined the width of this sharp line to be less than $0.3 \mathrm{~nm}$. Its position shifted by $4.5 \mathrm{~nm}\left(143 \mathrm{~cm}^{-1}\right)$ when the excitation wavelength was luned from $559 \mathrm{~nm}$ to $563.5 \mathrm{~nm}$. thus exactly corresponding to the energy difference of the excitation lasers. This fact clearly demonstrates the presence of the Raman gain in the DOO-PPV films.

In order to identify the active Raman mode participating in the optical amplification, we measured the off-resonance, spontaneous Raman scattering response of DOO-PPV at low excitation intensities. The resulting Raman scattering spectrum shown in Fig.9(c) reveals strong Raman active lines at $968,1171,1285$, and $1584 \mathrm{~cm}^{-1}$, respectively. The latter mode is the strongest and therefore is the most likely candidate to be amplified by the gain medium at high excitation intensities. Indeed, as seen in Fig.9(c), we obtained excellent spectral overlap between the positions of the Raman mode at $1584 \mathrm{~cm}^{-1}$ and the narrow line in Fig. $9(\mathrm{~b})$. This demonstrates that we may use luminescent $\pi$-conjugated polymer thin films as inexpensive laser Ramanshifters for spectroscopy ${ }^{38}$. 


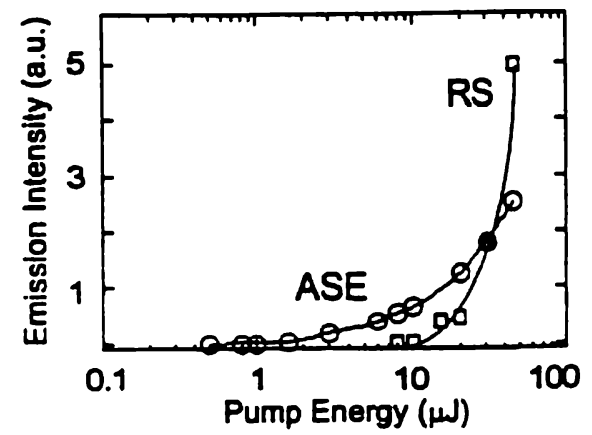

Fig. 10. Emission intensity vs. excitation pulse energy of the ASE band and amplified $1584 \mathrm{~cm}^{-1}$ Raman scattering (RS) mode in a DOO-PPV film for $563.5 \mathrm{~nm}$ excitation.

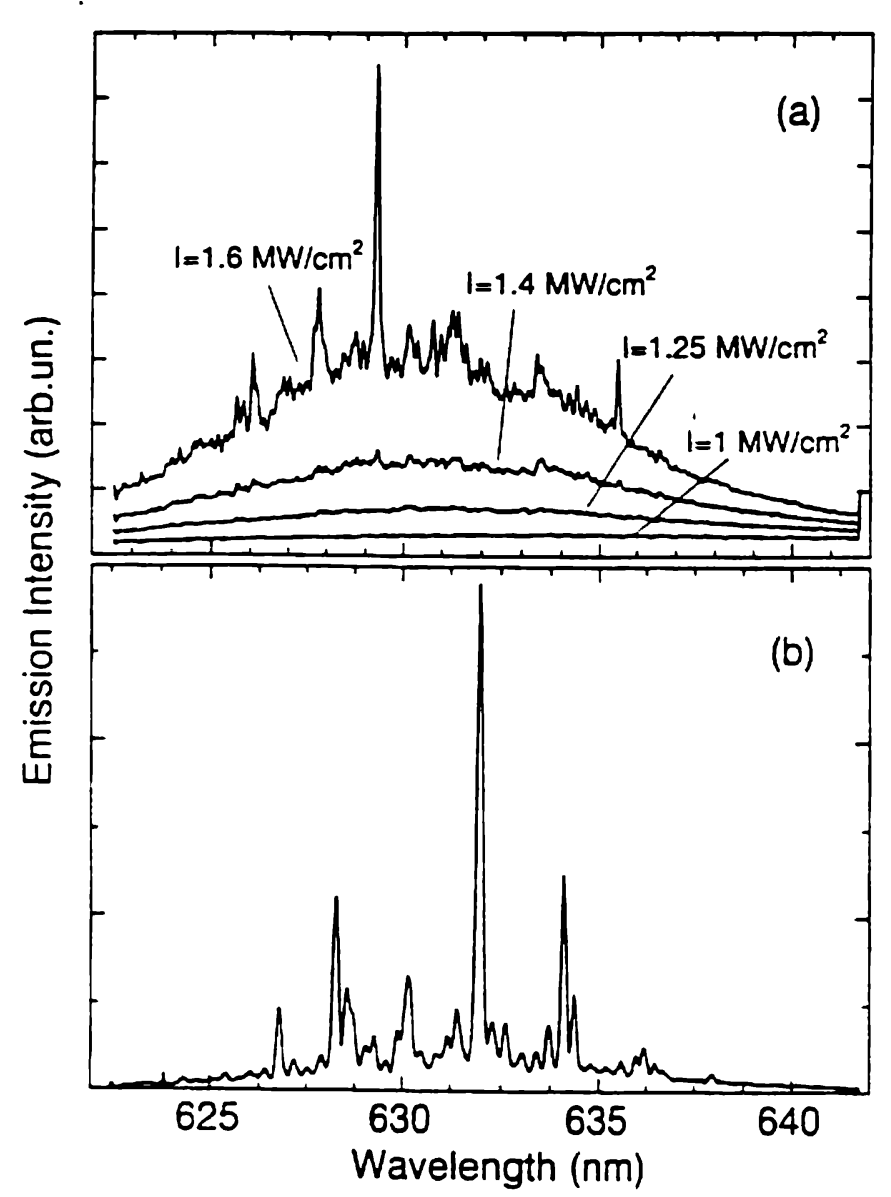

Fig Ii. Sumulated emission spectra of a DOO-PPV film obtained using a stripe-like excitation area with the length $L$ of $1 \mathrm{~mm}$ and width of $30 \mu \mathrm{m}$. (a) SE at different excitation intensilies, (b) SE at $I=2 \mathrm{MW} / \mathrm{cm}^{2}$ irom a different excited area of the same DOO-PPV film.

We measured the emission intensity vs. the excitation intensity for a DOO-PPV film excited at $563.5 \mathrm{~nm}$ (Fig.10). It is seen that the threshold excitation intensity for Raman gain is about one order of magnitude higher than that of the ASE, occurring at 8 and $0.5 \mu$. respectively. The resonant Raman amplification reveals, however. a higher slope efficiency compared to that of ASE. At the highest used excitation intensities it is therefore possible to generate emission where Raman scattering is dominant. In this case a narrow laser-like emission line is realized in the polymer films with no optical cavity. in addition to a residual. smaller and much broader ASE band. 


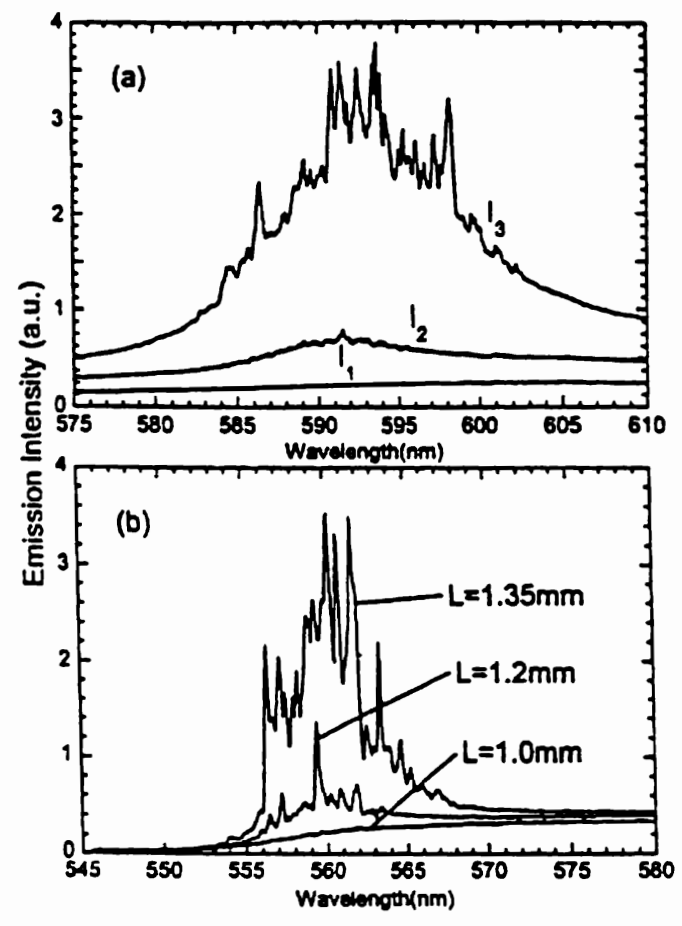

Fig.12. (a) SE spectra of a microcrystalline opal slab saturated with DOO-PPV solution, where $L=3 \mathrm{mr}, a=30 \mu \mathrm{m}, I_{1}=0.6$ $\mathrm{MW} / \mathrm{cm}^{2}, I_{2}=1 \mathrm{MW} / \mathrm{cm}^{2}$, and $I_{3}=2 \mathrm{MW} / \mathrm{cm}^{2}$. (b) SE spectra of an opal saturated with $\mathrm{R} 6 \mathrm{G}$ solution at various $L$ and cinstant $a=40 \mu \mathrm{m}$ and $I=1.5 \mathrm{MW} / \mathrm{cm}^{2}$.

\section{RANDOM LASING WITHIN POLYMER FILMS AND OPAL-PHOTONIC CRYSTALS}

In the absence of an optical cavity, a gain medium is expected to exhibit ASE, which is a stimulated emission process without a feedback mechanism that would otherwise lead to lasing. ASE typically results in emission spectral narrowing and exciton lifetime shorening. both of which have indeed been observed in thin polvmer films ${ }^{4}$. Our recent close examination of stimulated emission in polymer waveguides', however, have shown that the ASE model might not be adequate to fully describe the observed phenomena. In our measurements, we used the experimental apparatus already described in section 2. Thin DOOPPV films. spin-coated on nat quarz substrates, were photoexcited using an excitation area in the shape of a thin stripe with length $L$ and width $a^{19}$. The polymer emission was collected in the direction along the stripe axis; as reported previouslys. stumulated emission predominantly occurred in this direction.

Fig.11(a) illustrates typical emission spectral narrowing observed in one of the studied DOO-PPV films. Due to stimulated emission, a featureless band at $-630 \mathrm{~nm}$ with a characteristic bandwidth of $\sim 10 \mathrm{~nm}$ appeared at the threshold excitation intensity $I_{A}$. At $>I_{A}$ the spectral narrowing process continued and resulted in the formation of numerous, randomly positioned spectral lines with linewidths on the order of $0.1 \mathrm{~nm}$. The later process could be observed only at excitation intensities greater than a second threshold intensity $I_{\mathrm{B}}$ (see Fig.11), provided that the excitation stripe width was small $(a<100 \mu \mathrm{m})^{19}$. For $>I_{\mathrm{B}}$ the emission spectra were typically dominated by a few strong, "super-narrowed" lines, as shown in Fig.11(b). "The position and strength of these lines appear to be random and different for different excited areas of the same film. We note, however, that the phenomenon of spectral "supernarrowing" could not be seen unless a thin stripe-like excitation area was used".

Fig.12(a) and (b) show the spectral evolution of opal photonic crystals infiltrated with DOO-PPV and R6G solution, respectively, upon increasing the excitation intensity $l$. We see the onset of ASE at $I=I_{2}\left(=I_{\mathrm{A}}\right.$ ) (Fig.12(a)). However at $I=I_{3}>I_{\mathrm{B}}$ spectral narrowing due to ASE was followed by the development of a series of narrow spectral lines. R6G solution infiltrated into the opal crystals exhibited a similar effect, but in a slightly different spectral range corresponding to the maximum optical gain of R6G, as shown in Fig.12(b). In this case, however, instead of increasing $I$ at constant $L$, we achieved super-narrowing by increasing $L$ while keeping $I$ constant. Analogous measurements were done with DOO-PPV films and solutions ${ }^{19}$; these 
experiments showed that the magnitudes of both $I_{A}$ and $I_{B}$ decrease as $L$ increases. Such a behavior has beell observed before in ASE processes ${ }^{b}$ and distributed feedback (DFB) lasers ${ }^{13}$. We note that in these latter experiments we used bulky opal slabs, where there was no waveguide formation. We also note that in identical experimental conditions. SE from free-standing pure solutions of either DOO-PPV or R6G did not produce similar super-narrowing; this demonstrates that this effect is indeed due to light scattering inside the opals.

Laser action should require a resonant optical feedback mechanism, e.g. reflections from the sample edges. However, such reflections do not exist in neither the polymer films nor the opal slabs. Furthermore, the extreme sensitivity of the finely structured spectra to the exact position of the excitation area on the sample indicates that the structural resoriances must come from inside of the excited region. In this case the spectral super-narrowing is due to random feedback produced by light scattering in the bulk. However, whereas scattering centers can be easily identified in opals and gel-films as silica nanoballs, it is not clear at present what could play such a role in pristine DOO-PPV films.

It has been argued that very strong scattering is required for random lasing. In fact, lasing was predicted to occur only if the scattering length, $\Gamma$, is on the order of $\lambda^{33.40}$. In our case $\Gamma \gg \lambda$, even if we attribute $\alpha_{\text {ret }}$ of $-30 \mathrm{~cm}^{-1}$ solely to a scattering process. We speculate, however, that even weak scattering may produce sufficient optical feedback to cause the appearance of random resonances in the stimulated emission spectrum of a high gain medium. In case of thin stripe excitation this process may resemble the mechanism of distributed feedback ${ }^{19}$, in which we envision weak backscattering repeatedly occurring along the entire length of the excitation stripe. Such backscattering is speckle-like and therefore wavelength selective, where the selection process is largely random and determined by the structural arrangement of the scatterers inside the excitation volume.

\section{CONCLUSIONS}

We observed and studied various regimes of stimulated emission produced by optical pulsed excitation of $\pi$-conjugated polymer films. Multimode lasing was demonstrated in various cylindrical microcavities, including self-assembled microrings on optical fibers and metal wires, and patterned microdisks. Such microcavities were shown to possess a high $Q$ factor, limited by intrinsic losses of the polymer gain medium. This high $Q$ value, however, depends on the excitation pulseuidth and decreases for longer excitation pulses. Operating micro-LEDs were fabricated using microring and microdisks configurations with the goal to produce laser emission using current injection. In the absence of a laser cavity, thin polymer films were shown to exhibit two unusual regimes of stimulated emission. in which narrow lines appeared in the amplified spontaneous emission spectrum. One of these regimes was found to be due to amplified Raman scattering. whereas the other regime is associated with optical feedback induced by random light scattering inside the films. The latter regime also operative in opal photonic crystals. with no relation 10 the existing Bragg stop bands. It will be interesting to find another possible laser action in the opals, which is more directly related to the opal stop bands.

\section{ACKNOWLEDGMENTS}

We thank R. Polson and J.D. Huang for laboratory assistance. and M. DeLong and G. Levine for some if the DOO-PPV samples. This work was suppored in part by the NSF grant \#DMR 97-32820 the Intemational Joint Research Program of NEDO (Japan) and the Center for Advanced Fibers and Films al Clemson University supported by the ERC Program of the NSF. grant \# ERC 9731680

*Bell Laboratories, Lucent Technologies, Murray Hill, NJ 07974

** Sandia National Labs, PO Box 969, Livermore, CA 94551

***Cavendish Laboratory, Madingley Road, Cambridge CB3 OHE, UK 


\section{REFERENCES}

1. N. Tessier, G. J. Denton and R Friend, "Lasing of conjugated polymer microcavities," Nature (London) 382, pp. 695697, 1996.

2. F. Hide, M. A. Diaz-Garcia, B. J. Schwartz, and A. J. Heeger, "Laser emission from solutions and films containing semiconducting polymer and $\mathrm{Ti}_{2}$ nanoctystals," Chem. Phys. Lett., 256, pp. 424-430, 1996; Science, 273, pp. 1833$1836,1996$.

3. H.J. Brower, V.V. Krasnikov, A. Hilberer, and G. Hadziioannou, "Blue superradiance from neat semiconducting alternating copolymer films," Adv. Materials, 8, pp. 935-938, 1996; G. Gelink, J.W. Warman, M. Remmers, and D. Neher, Chem. Phys. Lett., 265, pp. 320-324, 1997.

4. S. V. Frolov, M. Ozaki, W. Gellerman, K. Yoshino and Z. V. Vardeny, "Cooperative emission in $\pi$-conjugated polymer thin films," Phys. Rev. Lett., 78, pp. 729-732, 1997.

5. X. Long, A. Malinowski, D.D.C. Bradley, M. Inbasekaran, and E.P. Woo, "Superfluorescence and lasing in conjugated polymers," Chem. Phys. Lett., 272, pp. 6-9, 1997.

6. G. J. Denton, N. Tessler, M. A. Stevens and R H. Friend, "Spectral narrowing in optically pumped PPV films, " $A d$. Mater., 9. pp. 547-551, 1997.

7. C. Zenz. W. Graupner, S. Tasch, G. Leising. K. Mullen and U. Scherf, "Blue green SE from a high gain conjugated polymer," Appl. Phus. Lett., 71, pp. 2566-2568. 1997.

8. S.V. Frolov, K. Yoshino and Z.V. Vardeny, "Cooperative and stimulated emission in PPV thin films and solutions," Phus. Rev. B, 57, pp. 9141-9147. 1998.

9. S. V. Frolov, M. Shkunov, K. Yoshino and Z.V. Vardeny, "Ring microlasers from conducting polymers," Phys. Rev. B. 56. pp. R4363-4366, 1997.

10 N.D. Kumar, J.D. Bhawalkar, P.N. Prasad. F.E. Karasz and B. Hu "Solid state tunable cavity lasing in PPV block copolvmer." Appl. Phus. Lett., 71, pp. 999-1001. 1997.

11. Y. Kawabe et al., "Whispering-gallery-mode microring laser using a conjugated polymer," Appl. Phys. Lett., 72, pp. $141-143,1998$.

12. G. Wegmann. H. Giessen. A. Greiner and RF. Mahr. "Laser emission from a solid conjugated polymer; gain, tunability and coherence," Phys. Rev. B, 57, pp. R+218-4221. 1998.

13 M. D. McGehee. el al., "Semiconducting polymer distributed feedback lasers." Appl. Phys. Lett. 72. pp. 1536-1538. 1998.

It S.V. Frolov et al.. "Cylindrical microlasers and LEDs from conducung polymers." Appl. Phus. Ler'. 72. pp. 18021804. 1998: ibid. 72. pp. 2811-2813. 1998.

15. M. Berggren. A. Dodabalapur, R.E. Slusher. and Z. Bao. "Light amplification in organic thin films using cascade energy transfer." Nature. 389. pp. 466-469, 1997, Appl. Phvs. Letl., 71. pp. 2230-2232. 1997; Ad'. Mater., 9. pp. 968970. 1997.

16. V.G. Kozlov: V. Bulovic. P.E. Burrows. and S.R. Forrest. "Laser action in organic semiconducting waveguide and double heterostructure devices," Nature. 389. pp. 362-364. 1997.

17 A. Fujii. S.V. Frolov. Z.V.. Vardeny, and K. Yoshino. "Polymer electroluminescent diodes with ring microcavity structure," Jpn. J. Appl. Phys., 37, No. 6B. pp. L740-742. 1998.

18 S.V. Frolovi Z.V. Vardeny. A.A. Zakhidov, and R.H. Baughman. "Laser-like emission in opal photonic crystals," Opiic.s Commun., 162. pp. 241-246. 1999.

19 S V Frolov: Z.V. Vardeny, K. Yoshino. A.A Zakhıdov. and R.H. Baughman. "Stimulated emission in high-gain organic media." Phvs. Rev. B. 59. pp. R5284-5286. 1999.

20 M.N. Shkunov. W. Gellermann. and Z.V. Vardeny. "Amplified RRS in conducting polymer thin films." Appl. Phys. Lell. 73. pp. 2878-2880. 1998.

21 Z.G. Soos and S. Etemad. Chem. Phys. Lett. 194. pp. 341-345. 1992.

22 S. V. Frolor. M. Liess. P.A. Lane. M. Ozaki. K. Yoshino, W. Gellermann, and Z.V. Vardeny, "Exciton dynamics in soluble PPV: lowards an ultrafast excitonic suitch." Phy's. Rev. Lett., 78, pp. 4285-4288. 1997.

23. N.T. Hamson. G.R. Hayes. R.T. Phillips. and R.H. Friend. "Singlet intrachain exciton generation and decay in PPV," Phus. Rev. Lett. 77, pp. 1881-1892. 1996. 
24. I. Gontia et al., "Excitation dynamics in disubstituted polyacetylene," Phys. Rev. Lett., 82, pp. 4058-4061, 1999, and refs. therein.

25. M. Yan, L.J. Rothberg, F. Papadimitrakopoulos, M.E. Galvin, and T.M. Miller, "Spatially indirect excitons as primary photoexcitations in conjugated polymers," Phys. Rev. Lett., 72, pp. 1104-1107, 1994.

26. K. Yoshino, K. Tada, M. Ozaki, A.A. Zakhidov, and R.H. Baughman, "The optical properties of porous opals infiltrated with organic molecules," Jpn. J. Appl. Phys., 36, pp. L714-717, 1997.

27. D. Moses, "High quantum efficiency luminescence from conducting polymer insolution: a novel polymer laser dye." Appl. Phys. Lett., 60, pp. 3215-3217, 1992.

28. H.-J. Brouwer, V.V. Krasnikov, A. Hilberer, J. Wildeman, and G. Hadziioannou, "Novel high efficiency copolymer laser dye in the blue wavelength region," Appl. Phys. Lett., 66, pp. 3404-3406, 1995; W. Holzer, A. Penzkofer, S.H. Gong, A. Bleyer, and D.D.C. Bradley, "Laser action in poly(m-phenylene-vinylene-co-2,5-dioctoxy-p-phenylenevinylene)," Adv. Mater., 8, pp. 974-977, 1996.

29. M. Yan, L. Rothberg, B.R. Hsieh, and R.R. Alfano, "Exciton formation and decay dynamics in electroluminescent polymers observed by subpicosecond stimulated emission." Phys. Rev. Lett., 49, No. 14, pp. 9419-9422, 1994.

30. A. Schuizgen, C. Spiegelberg, M.M. Morrell, S.B. Mendes. B. Kippelen. N. Peyghambarian, M.F. Nabor, E.A. Mash. and P.M. Allemand, "Near diffraction-limited laser emission, from a polymer in a high finesse planar cavity." Appl. Phys. Lett., 72. No. 3. pp. 269-271, 1998.

31. Y. Kawabe, C. Spiegelberg, A. Schulzgen, M.F. Nabor, B. Kippelęn, E.A. Mash, P.M. Allemand, M. KuwataGonokami. K. Takeda, and N. Peyghambarian. "Whispering gallery-mode microring laser uslı1g a conjugated polymer," Appl. Phys. Lett., 72. No. 2, pp. 141-143, 1998.

32. R.E. Slusher, A.F.J. Levi, U. Mohideen. S.L. MCCall, S.J. Pearton, R.A. Logan, "Threshold characteristics of semiconductor microdisk lasers," Appl. Phvs. Lett. 63. No. 10. pp. 1310-1312, 1993.

33. A. Yariv, Quantum Electronics, (John Wiley \& Sons. New York, 1989)

34. S.L. McCall, A.F.J. Levi. R.E. Slusher, S.J. Pearton. and R.A. Logan, "Whispering gallery mode microdisk lasers." Appl. Phvs. Lett. 60, No. 3, pp.289-291, 1992.

35. V. Bulovic. V.G. Kozlov, V.B. Khalfin, and S.R. Forrest. "Transform-limited. narrow-linewidth lasing action in organic semiconductor microcavities," Science. 279. pp. 553-555. 1998

36. S.V. Frolov, A. Fujii. D. Chinn. M. Hirohata. R. Hidayat. M. Taraguchi. T. Masuda. K. Yoshino, and Z.V. Vardeny. "Microlasers and micro-LEDs from disubstituted polyacetvlene," Adv. Mater., 10, No. 11, pp. 869-872, 1998

37 D.G. Lindzey. D.D.C. Bradley. S.F. Alvarado, and P.F. Seidler. "El from tunneling injection in PPV films," Nature, (london). 386, pp. 135-135, 1997.

38 M. Shkunov. W. Gellermann. and Z.V. Vardeny. University of Utah disclosure, 1997.

$39 \mathrm{~S}$ John. and G. Pang. "Theoñ of lasing in muluple-scattening medium." Phvs. Rev, A, 54, pp. 3642-3652, 1996.

4 D.S Wiersma. and A. Lagendijk. "Light diffusion with gain and random lasers." Phus. Rev. E, 54. pp. 4256-4265, 1996. 